Provided for non-commercial research and education use. Not for reproduction, distribution or commercial use.

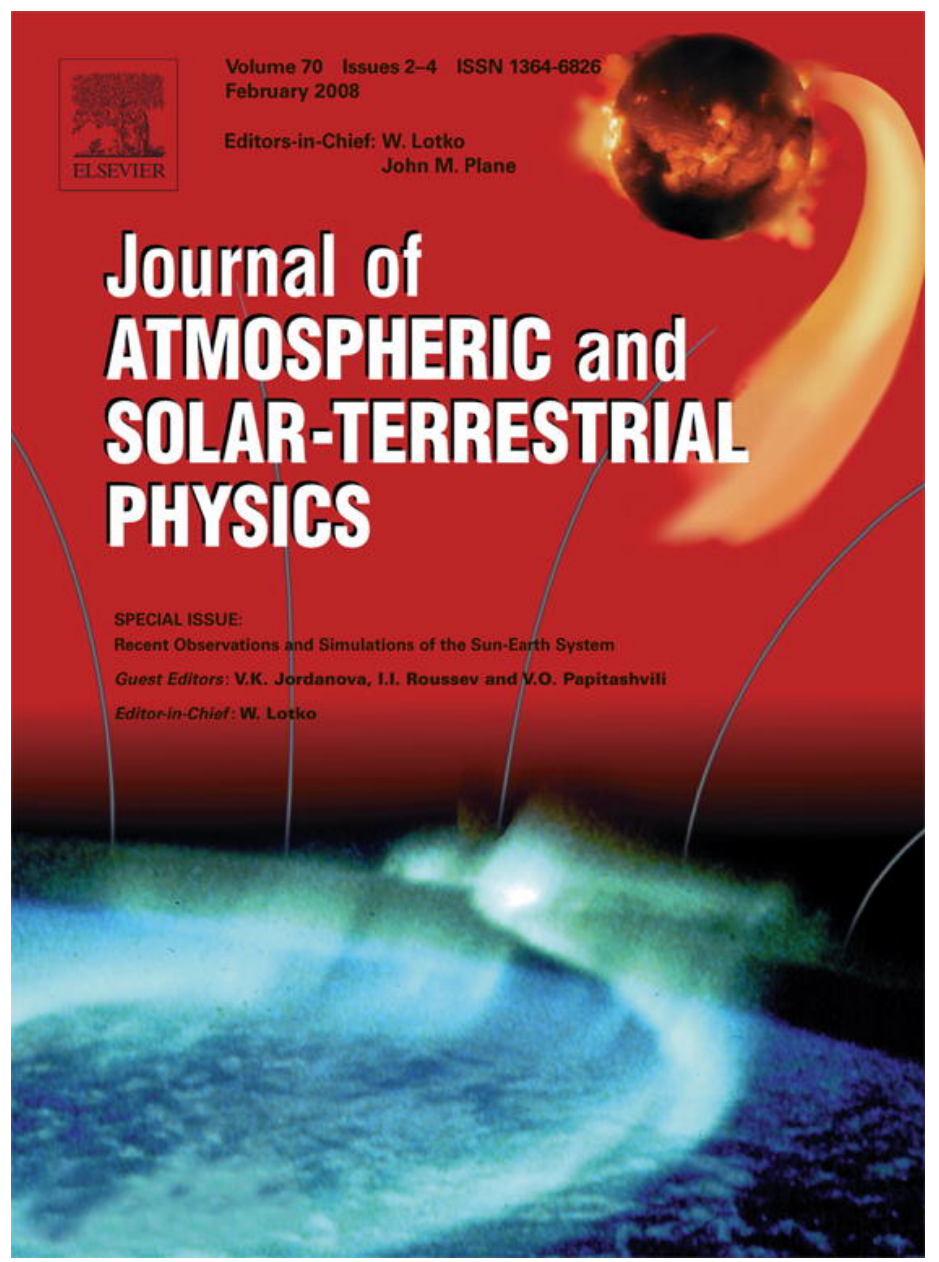

This article was published in an Elsevier journal. The attached copy

is furnished to the author for non-commercial research and education use, including for instruction at the author's institution, sharing with colleagues and providing to institution administration.

Other uses, including reproduction and distribution, or selling or licensing copies, or posting to personal, institutional or third party websites are prohibited.

In most cases authors are permitted to post their version of the article (e.g. in Word or Tex form) to their personal website or institutional repository. Authors requiring further information regarding Elsevier's archiving and manuscript policies are encouraged to visit: 


\title{
Formation of the delayed relativistic solar electrons
}

\author{
Ilan Roth \\ Space Sciences, University of California, Berkeley, CA 94720, USA
}

Accepted 27 August 2007

Available online 22 October 2007

\begin{abstract}
The existence of non-thermal electrons in the solar atmosphere and along the heliospheric field lines is deduced through emission of electromagnetic waves and via direct in situ measurements at $1 \mathrm{AU}$. The relation between the in situ electrons, their spectral shapes and the relative timing with respect to imaging and spectrographic observations are important in identifying potential acceleration sites and in understanding of the processes which control the formation of the delayed, (mildly) relativistic electrons which are observed in conjunction with flares or coronal mass ejections (CMEs). In contrast to previously suggested paradigms of acceleration of relativistic electrons around the CME shocks or at the flare site, it is suggested here that the delayed acceleration occurs along the stretched, closed coronal field lines, when an anisotropic seed population of low-energy electrons is injected in conjunction with the high frequency coronal radio bursts behind the large $\mathrm{CME}$, as recorded by radioheliographs. The energization proceeds as a bootstrap process due to interaction with oblique whistler waves. The flare serves mainly as a time reference for the electromagnetic emissions, while the propagating CME subsequently opens an access for the relativistic electrons to the interplanetary medium.
\end{abstract}

(C) 2007 Elsevier Ltd. All rights reserved.

Keywords: Solar electrons; Flares; CME; Radio waves

\section{Introduction}

Simultaneously observed electrons, which can be traced through the coronal electromagnetic emissions, and electrons observed through their propagation along the heliospheric magnetic field and measured in situ at $1 \mathrm{AU}$, impose constraints regarding emission processes operating in the corona and in the heliosphere. Various investigations of relative timings between radiation and particles, together with correlation between spectral wave distribution and electron energies/pitch angles indicate that often the electrons emerge at several injection forms. Although some very energetic flares

E-mail address: ilan@ssl.berkeley.edu display simultaneous sources of (near) relativistic electrons, as observed through Bremsstrahlung emissions, the present investigation is focused on the subset of measurements when the (near) relativistic electrons are observed at $1 \mathrm{AU}$ with a solar injection time significantly delayed with respect to the lower energy electrons, pointing out to different inception and energization mechanisms.

The time history and the energy of the measured electron fluxes at $1 \mathrm{AU}$ provide an important clue to the "injection" mechanism. Since the first observations of the impulsively accelerated electrons (Van Allen and Krimigis (1965); Anderson and Lin, 1966) it was confirmed that most of the impulsive electron events result in excitation of interplanetary (IP) type III emissions at the range of $30 \mathrm{kHz}-\mathrm{few} \mathrm{MHz}$ 
(Lin, 1985). Conversion of these frequencies into the local density shows that the excitations of type III occur from the vicinity of the solar surface all the way to $1 \mathrm{AU}$. More precise timing of the observed electron fluxes (Krucker et al., 1999) showed that the injected electrons could be characterized into two categories: low-energy which are injected almost instantaneously with the electromagnetic radiation and more energetic with a delay of 10-30 min. In a series of investigations Haggerty et al. (2003) found that the timing between the metric type III, hard X-rays (HXR), microwave bursts and $H_{\alpha}$ emissions implies delays of a fraction of second to few seconds, while the mildly relativistic fluxes at $30-350 \mathrm{keV}$ are delayed by up to $40 \mathrm{~min}$ with respect to the electromagnetic emissions. Klassen et al. (2005) investigated the intense Halloween 2003 event and found multiple electron populations: (i) low-energy type III electrons, (ii) higher energy near-relativistic, delayed $(10 \mathrm{~min})$ impulsive population and (iii) long-lasting high energy relativistic electrons with an onset of $25 \mathrm{~min}$ after the type III initialization. Bastian et al. (2001) showed distinctly in the 20 April 1980 event that non-thermal radio emissions could be interpreted only as synchrotron radiation due to spiraling $0.5-5 \mathrm{MeV}$ electrons around $1 \mathrm{G}$ magnetic field. Recent statistical analysis of the delay times between type III and $82 \mathrm{keV}$ injection (Kahler et al., 2007) indicates lack of consistent signature with a weak support for short, impulsive and long, shock correlated delays.

Due to the complicated nature of the emissions of these delayed electrons and the often-ambiguous observational correlation with flare, coronal or interplanetary shock, the determination of the acceleration process becomes elusive and abound into controversy. The suggested mechanism of cause-relation to these delayed events include a correlation between EIT/Morton waves or coronal shock as a mechanism of electron energization (Krucker et al., 1999; Klassen et al., 2002; Haggerty and Roelof, 2002), reconnection behind the coronal or coronal mass ejections (CME) shock (Klein et al., 2005; Maia and Pick, 2004), direct energization at the CME shock (Simnett et al., 2002) or particle propagation effects across the magnetic field lines (Cane and Erickson, 2003). Reiner and Kaiser (1999) showed that taking into account the observed timing and frequency drift rates, some intense flarerelated type II events observed by ground telescopes cannot be related to the CME-excited type II emissions observed by WAVES experiment on
WIND. They attributed this result to two distinct shocks, with different excitation mechanisms. All these models do not determine an explicit process which can address the energy and the time scale for the delayed electrons.

In this paper we sketch a scenario for a large postflare configuration and a mechanism for electron energization to relativistic energies, as well as a partial yardstick for observation of the relativistic electrons.

\section{Description of the model}

Energization of charged particles and formation of non-thermal populations in magnetized plasma can be viewed on the single particle level as a violation of one or more of the adiabatic invariants. In the presence of an inhomogeneous magnetic field with partially closed field lines and local minima in the magnetic field strength the electrons conserve several invariants as they gyrate around, bounce along and drift across the magnetic field.

The suggested scenario progresses as follows: at a post-flare period, after CME uplift, strong spectroscopic burst signals at frequencies of several hundreds $\mathrm{MHz}$ are observed by radioheliographs, indicating low-coronal reconfiguration without direct correlation to the propagating CME (e.g. Maia and Pick, 2004). These bursts are not related to the flare event and occur at separate sites, usually at large distances from the original flare. It is therefore conjectured, similarly to the in situ observations at the active terrestrial magnetotail, that coronal bursts behind the CME inject low-energy, nonisotropic electrons. These electrons drift across the perturbed, inhomogeneous coronal field and destabilize whistler waves. As such, the process may be viewed as a bootstrap: the free magnetic energy is partly converted into nonisotropic electron energy; the injected electrons excite electromagnetic waves which pitch angle diffuse the bulk and energize the tail of their distribution. The energization proceeds via resonant interaction between the waves and the electrons: the parameter space of these resonances increases significantly when the waves acquire a perpendicular wavenumber, which generally occurs as the wave propagates along the increasing magnetic field and denser plasma, as simulated often at the Earths magnetosphere (e.g. Thorne and Horne, 1994; Bortnik et al., 2003). Therefore, the (near) relativistic electrons are not energized in 
flares or by the CME shocks. The role of the CME itself is only indirectly related to the acceleration process: (1) as it emerges from the solar atmosphere it leaves behind an unstable coronal configuration which erupts either as a short, large event or intermittently over tens of minutes, (2) it reconnects the closed coronal with the IP field lines, allowing access to the interplanetary medium.

The above-mentioned process relies on the effectiveness of the resonant interaction between the electrons and the whistler waves. Both the waves and the electrons bounce along the inhomogeneous field lines: adiabatic electrons increase their pitch angles as they enter the more intense magnetic field while the whistler waves modify their wavenumbers as they follow their ray-tracing trajectories with changing derivatives of the dielectric tensor. Direct derivation from the linearized Vlasov equation results in a perturbed distribution $\delta f\left(v_{\|}, z, \rho, t\right) \sim$ $J_{n}\left(k_{\perp} \rho\right) \cos \left[k_{\|} z-(\omega-n \Omega / \gamma) t+\psi_{\omega}\right]$ for an electron with gyroradius $\rho$, gyrofrequency $\Omega$, parallel velocity $v_{\|}$along the local magnetic field in the $z$ direction and relativistic factor $\gamma=\left(1-v^{2} / c^{2}\right)^{-1 / 2}$, interacting with a wave of frequency $\omega$, wavenumber $\left(k_{\|}, k_{\|}\right)$and phase $\psi_{\omega}$, indicating that as an electron and a whistler propagate along the magnetic field lines, modifying their pith angle/ energy and wavenumber/amplitude, respectively, they may encounter numerous locations where the phase of the perturbations is almost stationary, resulting in the resonance condition: $k_{\|} v_{\|}=\omega-$ $n \Omega / \gamma$. Landau resonance is obtained when $n=0$, while the standard whistler resonance, the only one that applies to electrons with very low perpendicular energy $\left(k_{\perp} \rho \sim 0\right)$ is satisfied for $n=-1$, i.e. when the waves and the electrons move antiparallel to each other. Since the Bessel functions $J_{n}$ are nonnegligible only when their argument reaches values of a fraction of the integer $n$, the interaction with higher resonances increases with the energy/gyroradius. The interaction then is satisfied by all available negative and positive (anomalous) integers. Therefore, once a tail is formed in the distribution function its energization rate is increasing due to the large number of wave resonances, decreasing substantially the energization time scale. At each interaction the stochastic process modifies the energy of the electron, depending on the value of the relative phase $\psi_{\omega}$, however, the only important modifications in the distribution are due to the electrons whose energy is resonantly increased. The time scale depends on the amplitude of the waves and on the "density" of resonant locations for the bouncing electrons.

The simulation model follows test particles in the presence of an inhomogeneous magnetic field and oblique whistler waves. The stretched magnetic field is approximated by a slowly changing magnetic field

$B(x, y, z)=B_{0}\left[1+z(z \hat{z}-x \hat{x}-y \hat{y}) / D^{2}\right]$,

where $z$ denotes the field axis, $D$ denotes the scale length of the magnetic field and $B_{0}$ is its minimum. The form (1) satisfies $\operatorname{div} B=0$.

The wave electric field is given by $\left[E_{x} \cos \psi\right.$, $\left.E_{y} \sin \psi, E_{z} \cos \psi\right]$ with $E_{x}=E_{0}$ denoting the electric wave amplitude, the wave phase $\psi=\left[\int \mathbf{k}(\varepsilon z)\right.$ $\mathrm{d} \mathbf{x}-\omega t+\phi]$, where $\phi$ is an arbitrary phase; the magnetic wave components are obtained from Faraday's law and the $\varepsilon$ functional dependence emphasizes the slowly changing wavenumber $\mathbf{k}=$ $(k \sin \theta, 0, k \cos \theta)$ along electron trajectory. Parallel propagation involves an electromagnetic, righthand polarized mode, while oblique propagation makes the polarization elliptical and adds an electrostatic component. In the cold limit the square of the refractive index $c^{2} k^{2} / \omega^{2}$ is given by $\left[B-\left(B^{2}-4 A C\right)^{0.5}\right] / 2 A$, with

$A=\varepsilon_{1} \sin ^{2} \theta+\varepsilon_{3} \cos ^{2} \theta$

$B=\varepsilon_{1}\left(\varepsilon_{3}+A\right)-\varepsilon_{2}^{2} \sin ^{2} \theta$,

$C=\varepsilon_{3}\left(\varepsilon_{1}^{2}-\varepsilon_{2}^{2}\right)$,

where $\varepsilon_{i}$ denote the components of the dielectric tensor. The wavenumber changes along the magnetic field due to varying dielectric tensor.

The variables which describe the evolution of the system include particle phase space and wave phase $\psi_{\omega}$, which changes explicitly with the elapsed time, the time-dependent particle position and the location-dependent wavenumber. Explicitly, we solve the following set of equations:

$\mathrm{d} \mathbf{x}(t) / \mathrm{d} t=\mathbf{v}(t)$,

$\mathrm{d} \mathbf{p}(t) / \mathrm{d} t=(q / m)[\mathbf{E}[x(t)]+\mathbf{v} \times \mathbf{B}[x(t)] / c]$,

$\left.\mathrm{d} \psi_{\omega}(t) / \mathrm{d} t=k_{\|}(z) v_{\|}(t)\right]+k_{\perp}(z) v_{x}(t)-\omega$.

$\mathbf{B}(x)$ includes the guide field (Eq. (1) as well as wave fields. We integrate the relativistic electron equation of motion together with the wave phase and follow the non-adiabatic changes whenever the electron enters a resonance along its trajectory.

Fig. 1 shows the calculated square of energy change $\left(W-W_{0}\right)^{2}$ during $80 \mathrm{~s}$ of simulation of few 


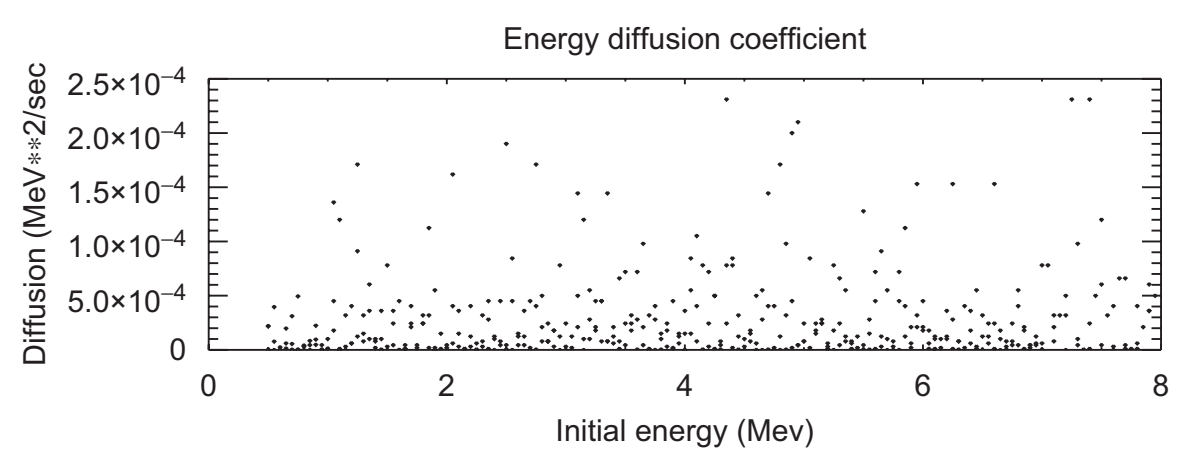

Fig. 1. Energy diffusion as a function of the initial electron energy.

hundred electrons, as a function of their initial energy $W_{0}$. The chosen wave amplitude is $2 \mathrm{mV} / \mathrm{m}$ with a wavenumber at magnetic minimum inclined by $30^{\circ}$ with respect to the magnetic field. One observes a statistically homogeneous energy diffusion with a small increase as a function of the initial electron energy. Since the electron diffusion increases quadratically with the electric field amplitude, wave field of $10 \mathrm{mV} / \mathrm{m}$ will result in energy diffusion of (fraction of) $\mathrm{MeV} /$ minute.

\section{Discussion and summary}

The in situ observation of relativistic or nearrelativistic electrons at $1 \mathrm{AU}$ with a detailed extrapolation to their release time at the solar atmosphere often poses a puzzle regarding the mechanism of their formation and the relevant magnetic configuration. Numerous measurements confirmed that in a large percent of events the energetic electrons begin their journey from the solar environment to the observing satellites at $1 \mathrm{AU}$ with a significant delay with respect to the main stage of the intense flare, the start of the type III emissions, or the initiation of the coronal mass ejection uplift. In this subset of observations the hard X-rays and the high frequency radio waves $(300 \mathrm{MHz})$ occur at time delays of less than $1 \mathrm{~s}$ (Aschwanden et al., 1995), the microwave and HXR show delays up to tens of seconds (Silva et al., 2000), while the (quasi) relativistic electrons are injected into the open field lines tens of minutes later. The experimental conclusion in these cases (e.g. Wang et al., 2006) favors two or more distinct electron populations with different acceleration and injection mechanisms. The source of the delayed relativistic electrons remains controversial, requiring consistency in the post-flare configuration between the particle and the electromagnetic observations.

Acceleration models of electrons to relativistic energies due to the standard mechanisms of CMEdriven shocks or magnetic reconnection encounter difficulty due to their small gyroradii which ties them to the magnetic field and makes them often impervious to major violations of adiabatic invariants. Co-rotating interaction regions (CIR) detect electrons with energies up to $380 \mathrm{keV}$ (Keppler et al., 1995), although rarely and mainly at low heliographic altitudes; in a vast majority of events no significant electron spike is observed in association with a passing CME shock. Terrestrial magnetotail substorms inject $\sim 100 \mathrm{keV}$ electrons (Birn et al., 1998) and some very intense flares produce Bremsstrahlung emission up to relativistic energies (Krucker et al., 2007); kinetic models include (a) intense Alfvenic turbulence (Miller et al., 1996, 1997) along the coronal field lines, and (b) Fermilike acceleration (Drake et al., 2006) during contraction of elliptical magnetic islands in the reconnection ion diffusion region (as observed in simulations, but not confirmed yet experimentally). However, the flare-related, weak reconnection events rarely result in a formation of relativistic distributions. Therefore, it is a plausible conclusion that flares and CMEs are often mainly auxiliary players in the formation of the delayed relativistic electrons, and the required mechanism for a fasttime violation of the first or second adiabatic invariants is due to coherent electromagnetic waves. Natural candidates are whistler waves which are known to propagate and reflect along the inhomogeneous field lines; the source of these waves is mostly due to low-energy anisotropic electrons, which are ejected in conjunction with the observed coronal bursts. At the terrestrial magnetosphere whistlers are excited at the recovery phase of 
terrestrial magnetic storm, when a distant, magnetically unstable magnetotail injects non-isotropic, low-energy electrons into low L-shells (measuring equatorial distance in units of terrestrial radii); the correlation between substorms and the ducted whistler waves has been shown as a valid energization mechanism for the radiation belt electrons (Meredith et al., 2003; Horne and Thorne, 2003).

The proposed scenario of an active corona includes a local flare reconfiguration resulting in $\mathrm{X}$-rays, synchrotron and radio emission and a large scale release of magnetic energy with an uplift of CME. The flare itself serves mainly as a time reference for the electromagnetic emissions, while the CME forms the environment susceptible to bursts and the opening gate for the release of the energetic electrons, respectively. Large CME leaves behind magnetic field in a marginally stable configuration, including numerous magnetically unstable sites. These sites, some of them at large distances from the flaring site, become then the sources of the observed high frequency radio bursts. The bursts release magnetic energy in the CME evacuated domain, in analogy to terrestrial substorms. Nancay Radioheliograph (NRH) observations indicate clearly that the bursts occur at the corona, mostly without connectivity to the flare site, and behind the intense propagating CME (Pick and Maia, 2005). The outcome of these bursts results in a filling of the closed flux tubes with anisotropic, low-energy electrons which excite whistler waves while drifting across the field lines; these waves interact with the tail of the electron distribution energizing it promptly, forming an $\mathrm{MeV}$ tail over several minutes. The incubation time for the energization depends on the amplitude of the waves and on the number of resonant encounters between the electrons and the waves. The amplitudes of the whistler waves at the solar environment are much higher than in the magnetosphere since they scale approximately with the more intense background magnetic field. Additionally, the terrestrial low-L magnetic field forms due to dynamo currents at the Earth's core, while the deformed coronal field after the CME lift-off becomes quite distorted and extended; the resulting gradients in magnetic field may allow more frequent resonant interactions, decreasing significantly the energization time scale. Although the energization of a single electron to relativistic energies is quite significant, the number of accelerated particles is minuscule with respect to the number of the whistler-exciting electrons and the energy gain of the relativistic electrons is significantly smaller than the whistler wave energy. The connectivity to the external open field lines is controlled by the propagating CME as it reconnects the open interplanetary field lines with the closed coronal lines, allowing access of energetic particles to the IP medium. When the CME loses its connectivity to the corona during its expansion into the IP medium, the relativistic electrons should not be observed in the IP medium, hence a significant decrease in their fluxes may be construed as an indicator of the changing structure of the propagating CME.

One may, conversely, state that the delayed relativistic electrons will not be observed without a major reconfiguration leading to an ejection of an intense CME. The condition for the formation of the seed population requires numerous coronal emissions at high $(100 \mathrm{~s} \mathrm{MHz})$ frequencies behind the propagating CME. This condition forms a partial yardstick for observation of the delayed, solar relativistic electrons. These electrons become, therefore, an important feature of major coronal perturbations. They may also serve as a crucial link in the Sun-Earth connection processes, forming the required outer magnetospheric phase space density, which enhances the radiation belt via bethatron mechanism (violation of the third invariant) during terrestrial magnetic storms.

\section{Acknowledgments}

The author thanks the referees for their constructive comments and expresses his gratitude to the Max Planck Institute for Solar System Research in Katlenburg-Lindau, Germany, for the hospitality during the final phase of this research. This work was supported in part by NASA Grants NNG05GC72G and NNG05GL27G.

\section{References}

Anderson, K.A., Lin, R.P., 1996. Physical Review Letters 16, 1121.

Aschwanden, et al., 1995. Astrophysics Journal 440, 394.

Bastian, T.S., Pick, M., Kerdraon, A., Maia, D., Vourlidas, A., 2001. Astrophysics Journal 558, L65.

Birn et al., 1998. Journal of Geophysical Research 103, 9235.

Bortnik, J., Inan, U.S., Bell, T.F., 2003. Journal of Geophysical Research 108, 1030.

Cane, H.V., Erickson, W.C., 2003. Journal of Geophysical Research 108, 1203. 
Drake, J.F., Swisdak, M., Che, H., Shay, M.A., 2006. Nature $443,553$.

Haggerty, D.K., Roelof, E.C., 2002. Astrophysics Journal 579, 841.

Haggerty, D.K., Roelof, E.C., Simnett, G.M., 2003. Advances in Space Research 32, 2673.

Horne, R.B., Thorne, R.M., 2003. Geophysical Research Letters $30,1527$.

Kahler, S.W., Aurass, H., Mann, G., Klassen, A., 2007. Astrophysics Journal 656, 567.

Keppler, E., et al., 1995. Space Science Reviews 72, 285; Science, 268, 1013

Klassen, A., et al., 2002. Astronomy and Astrophysics 385, 1078.

Klassen, A., et al., 2005. Journal of Geophysical Research 110, A09S04.

Klein, K.L., Krucker, S., Trottet, G., Hoang, S., 2005. Astronomy and Astrophysics 431, 1047.

Krucker, S., Larson, D.E., Lin, R.P., Thompson, B.J., 1999. Astrophysics Journal 519, 864.

Krucker, S., White, S.M., Lin, R.P., 2007. Astrophysical Journal 669, L49.

Lin, R.P., 1985. Solar Physics 100, 519.
Maia, D.J.F., Pick, M., 2004. Astrophysics Journal 609, 1082.

Meredith, N.P., Horne, R.B., Thorne, R.M., Anderson, R., 2003. Geophysical Research Letters 30, 1871.

Miller, J.A., Larosa, T.N., Moore, R.L., 1996. Astrophysical Journal 461, 445.

Miller, J., et al., 1997. Journal of Geophysical Research 102, 12631.

Pick, M., Maia, D.J.F., 2005. Advances in Space Research 35, 1876.

Reiner, M.J., Kaiser, M.L., 1999. Geophysical Research Letters $26,397$.

Silva, A.V.R., Wang, H., Gary, D.E., 2000. Astrophysical Journal 545, 1116 .

Simnett, G.M., Roelof, E.C., Haggerty, D.K., 2002. Astrophysics Journal 579, 854.

Thorne, R.M., Horne, R.B., 1994. Journal of Geophysical Research 99, 17249.

Van Allen, J.A., Krimigis, S.M., 1965. Journal of Geophysical Research 70, 5737.

Wang, L., Lin, R.P., Krucker, S., Gosling, J.T., 2006. Geophysical Research Letters 33, L03106. 